\title{
Caracterização de concretos refratários projetáveis para o revestimento de calcinadores de alumina
}

\section{(Characterization of alumina fluid-flash calciner refractory castable lining)}

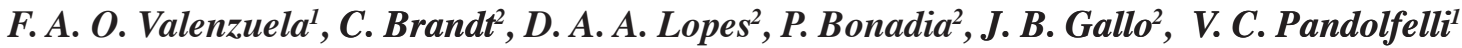 \\ ${ }^{I}$ Grupo de Engenharia de Microestrutura de Materiais- GEMM \\ Departamento de Engenharia de Materiais, Universidade Federal de S. Carlos \\ Rod. Washington Luiz, km 235, C.P. 676, S. Carlos, SP 13565-905 \\ ${ }^{2}$ Departamento de Aplicações e Desenvolvimento, Alcoa Alumínio S.A. \\ Rod. Poços-Andradas, km 10, Poços de Caldas, $M G$ \\ valenzuela@iris.ufscar.br,vicpando@power.ufscar.br
}

\begin{abstract}
Resumo
Diversos fatores interferem no desempenho e na vida-útil do revestimento refratário em unidades de calcinação de alumina. O desconhecimento das melhores práticas de seleção e qualificação dos materiais para esta aplicação foi a força motriz para este trabalho. Foram caracterizados dois materiais comerciais aplicados por projeção (um via wet-shotcrete e outro por dry-gunning), indicados para o revestimento refratário dos vasos e dutos de calcinadores de alumina. Tais técnicas são importantes, uma vez que reduzem o tempo de aplicação e o lucro cessante da unidade. Os materiais em análise foram submetidos a baterias de testes laboratoriais, os quais seguem normas técnicas internacionais. Os ensaios foram divididos segundo a representatividade das solicitações que ocorrem no revestimento em operação, e os resultados obtidos foram usados para avaliar o seu potencial desempenho. O embasamento técnico e científico utilizado neste trabalho pôde orientar o desenvolvimento, a seleção e a aplicação de novas cerâmicas refratárias para o revestimento interno de calcinadores, com o objetivo de elevar a vida-útil, a disponibilidade e a confiabilidade das unidades, possibilitando diminuir o custo com manutenções.

Palavras-chave: concretos refratários projetáveis, calcinador de alumina, propriedades termomecânicas.
\end{abstract}

\begin{abstract}
Several aspects are important to define the working life of refractory lining for alumina calcinations units. The lack of knowledge of the best selection practices and qualification of the materials for this application have been the driving force of this work. Two commercial materials applied by projection (wet-shotcrete and dry-gunning) were characterized. Both are usual standards of refractories for lining vessels and pipes of alumina fluid-flash calciners. All the materials analyzed were submitted to laboratorial tests, which follow international technical standards. The tests were divided in indispensable, necessary and occasional, according to the importance of the solicitations of the refractory lining under operation. The technical and scientific knowledge applied in this work can guide the development, the selection and the application of new refractory castables for the internal lining of alumina fluid-flash calciners, increasing their working life, productivity and the reliability of the units, and reducing the maintenance costs.

Keywords: projected refractory castables, alumina fluid-flash calciner, thermomechanical properties.
\end{abstract}

\section{INTRODUÇÃO}

Desde 1950 os maiores fabricantes de alumínio do mundo começaram a produzir a alumina do processo Bayer-HallHèroult em calcinadores de leito fluidizado, seja tipo Lurgi (sem vaso de retenção-HV) ou tipo Mark (com HV) $[1,2]$.

$\mathrm{O}$ uso de calcinadores verticais tornou o processo termicamente mais eficiente, com maior produtividade e com menores custos de operação $[1,3]$. Os atuais calcinadores de leito fluidizado podem atingir $1500 \mathrm{t} / \mathrm{dia}[4,5]$, com uma economia de combustível de até $40 \%$, em relação a outros processos de calcinação [6]. Mas há uma dificuldade natural de controlar o processo devido às diversas variáveis que atuam simultaneamente, tais como pressões parciais, altas temperaturas, carga erosiva e altas velocidades de transporte [5].

O coração do calcinador de alumina (Fig. 1) de leito fluidizado é o vaso principal do forno. $\mathrm{O}$ ar de combustão é pré-aquecido e entra na base do vaso sendo misturado com o combustível que é injetado nos queimadores. Quando a combustão ocorre, a ascensão dos gases gera uma corrente convectiva com velocidade alta o suficiente para que o hidróxido que está sendo alimentado se mantenha em 


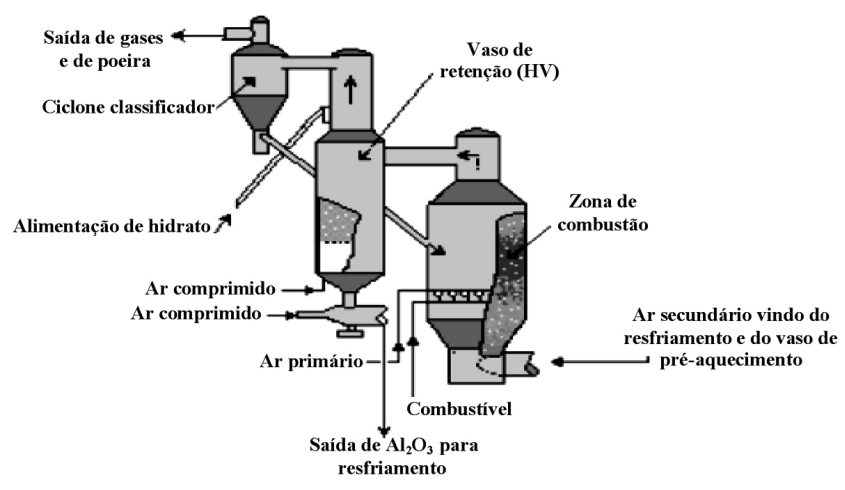

Figura 1: Ilustração de um calcinador de leito fluidizado tipo Mark (com HV) [3].

[Figure 1: Schematic drawing of Mark's fluidized-bed alumina flash calciner.]

suspensão no fluxo de gases e possa ser conduzido do vaso principal para os próximos estágios da calcinação, como a retenção, classificação e resfriamento [1,5].

Durante a operação normal da unidade, o revestimento refratário dos calcinadores sofre solicitações termomecânicas como a erosão a quente em virtude do impacto das partículas em suspensão, e a fluência das âncoras e do revestimento ocasionado pelas ações simultâneas da força peso, da temperatura e do tempo $[4,6]$. No entanto, solicitações térmicas também acabam ocorrendo durante paradas de emergência, onde $\mathrm{o}$ resfriamento $\mathrm{e} \mathrm{o}$ aquecimento são, geralmente, acelerados em relação ao procedimento usual $[7,8]$.

Como em condições normais de operação o principal mecanismo de desgaste que deve ser considerado é a erosão, Le Page [3] realizou o modelamento da distribuição de sólidos durante a calcinação em escala laboratorial, utilizando um algoritmo de Euler-Lagrange. Este procedimento gerou dados da concentração volumétrica e da trajetória individual das partículas. A análise destes resultados indicou alguns padrões de fluxo específicos [8-10]. O volume do material alimentado sobe rente às paredes em um movimento circular turbulento. Em regiões do cachimbo, onde se observam altas velocidades
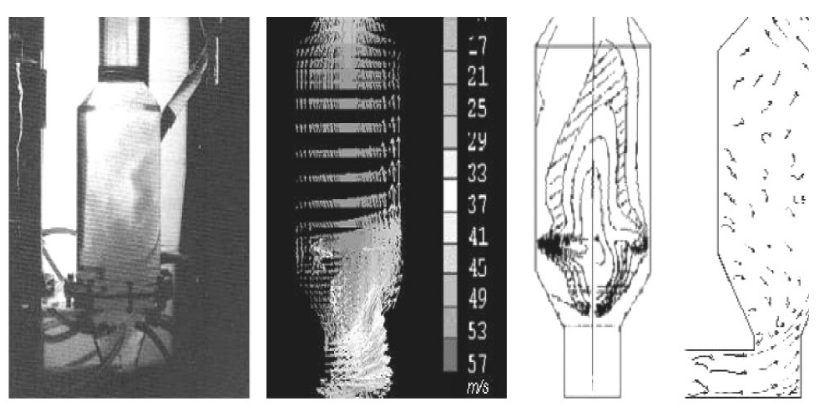

Figura 2: Simulação do campo de velocidades responsável pela erosão dos refratários em um calcinador de alumina de leito fluidizado $[3,10,12]$.

[Figure 2: Simulation of erosion velocity field to the refractory lining in the fluidized-bed alumina flash calciner.] de fluxo (até $55 \mathrm{~m} / \mathrm{s}$ ), áreas com alta taxa de erosão no revestimento refratário são verificadas (Fig. 2) [6, 11, 12].

Todas as preocupações que envolvem a melhoria de desempenho dos calcinadores se devem ao fato do processo de calcinação interferir seqüencialmente sobre as etapas da produção de alumínio primário, pois as propriedades físicoquímicas das partículas de alumina afetam a qualidade do produto final, o alumínio, bem como o consumo de energia elétrica das células Hall-Hèroult $[6,9]$.

Dentre as diversas propriedades da alumina que o processo de calcinação pode afetar, as mais relevantes são a elevação do teor de $\mathrm{SiO}_{2}$ pelo desgaste do revestimento refratário, o aumento na concentração de finos $(<45 \mu \mathrm{m})$ que pode gerar excesso de poeira na sala de cubas, e a redução da área superficial específica, que possui relação com a reatividade da alumina e com sua solubilidade na criolita [8].

Por todas estas importantes influências, a força motriz para este trabalho foi o desenvolvimento de melhores práticas de seleção e qualificação de materiais refratários para calcinadores de alumina em leito fluidizado, juntamente com outros passos constituintes da abordagem sistêmica utilizada para aumentar o desempenho dos calcinadores, realizadas por parceiros industriais, tais como: a) análise e melhoria do projeto da unidade de calcinação, b) instalação correta e otimizada dos refratários, c) tratamento térmico segundo curvas pré-estabelecidas, d) minimização de choques térmicos durante partidas e paradas, e) realização periódica de inspeções para verificar falhas no equipamento. Observando estes passos, será possível minimizar os custos com manutenção e elevar a confiabilidade dos calcinadores de alumina.

\section{MATERIAIS E MÉTODOS}

Devido a necessidade cada vez maior de elevar a disponibilidade de equipamentos imprescindíveis para a cadeia produtiva do alumínio, tal como o calcinador, e simultaneamente melhorar a confiabilidade das unidades, foram selecionados dois materiais de empresas brasileiras, os quais constituem as melhores tecnologias disponíveis para revestimento dos vasos e dutos de calcinadores.

Apesar dos concretos vertidos serem utilizados tradicionalmente por gerar menores porosidades e melhores homogenidades microestruturais, o que eleva o desempenho em serviço, demandam maior tempo para instalação e secagem [8].

Por isto, ambos os materiais propostos para esta caracterização são projetáveis. As amostras para os testes foram produzidas segundo a norma ASTM C903. O material chamado AWS foi aplicado por projeção a úmido (wet-shotcrete), que requer menor tempo de instalação que o vertido, mantendo uma boa homogenidade da microestrutura. Porém seu custo é superior em virtude da complexa logística de aplicação.

Já o material BDG foi projetado a seco (dry-gunning), o que requer um menor tempo de instalação com custos inferiores, mas gera uma microestrutura menos homogênea e mais porosa. 
Todos os materiais em análise foram submetidos a baterias de testes laboratoriais, os quais seguiram normas técnicas da ASTM (American Society for Testing and Materials) reconhecidas internacionalmente. Os ensaios foram divididos em três grupos: imprescindiveis, necessários e eventuais, segundo a representatividade das solicitações que ocorrem no revestimento em operação, e serviram para classificar o seu potencial desempenho (Tabela I).

No ensaio de erosão com $\mathrm{SiC}$ na temperatura ambiente foi seguida a norma ASTM C704. O ângulo de incidência do abrasivo em relação aos corpos foi de $90^{\circ}$, a distância da amostra ao bico ejetor foi de $203 \mathrm{~mm}$, a pressão do ar igual a $53,2 \mathrm{kPa}$ e a duração do teste de $450 \mathrm{~s}$. Para cada temperatura selecionada $\left(600,800,1000\right.$ e $\left.1250^{\circ} \mathrm{C}\right)$, quatro lajotas 114 x 114 x $30 \mathrm{~mm}^{3}$ foram pré-queimadas a $5{ }^{\circ} \mathrm{C} / \mathrm{min}$ com 5 $\mathrm{h}$ de patamar. $\mathrm{O}$ equipamento utilizado foi um erosímetro Solotest adaptado para desgaste de materiais refratários.

$\mathrm{O}$ teste de fadiga térmica utilizado neste trabalho é adaptado da norma ASTM C1171 e consiste de 10 ciclos térmicos com 15 min de residência a $1000{ }^{\circ} \mathrm{C}$, seguido de resfriamento súbito ao ar por $15 \mathrm{~min}$. $\mathrm{O}$ sistema utilizado consiste de um forno Lindberg Blue e de uma bandeja de alumínio revestida com alumina eletrofundida branca grão $8 \mathrm{~mm}$. Neste recipiente são colocados os corpos em resfriamento, os quais são mantidos em uma sala sem convecção de ar. Foram selecionadas as temperaturas de préqueima de 1000 e $1250{ }^{\circ} \mathrm{C}$, sendo utilizadas 10 amostras 152 x 25 x $25 \mathrm{~mm}^{3}$ para cada condição. Cinco foram submetidas aos ciclos térmicos e as outras 5 foram reservadas para a medida do módulo de ruptura sem dano por choque térmico. A avaliação da resistência ao choque térmico é realizada por meio da análise da resistência mecânica antes e após o ensaio térmico, associado com o valor absoluto residual da medida do módulo de ruptura por flexão em três pontos segundo a norma ASTM C133, com distância entre apoios de $125 \mathrm{~mm}$, rolete de apoio de $5 \mathrm{~mm}$ de diâmetro e uma taxa de carregamento de $0,772 \mathrm{kN} / \mathrm{min}$, realizado em uma máquina universal de ensaios mecânicos MTS, modelo 810/ TestStarII.

O módulo de ruptura a quente foi realizado segundo a norma ASTM C583. Foram usadas 5 amostras $150 \times 25 \times$ $25 \mathrm{~mm}^{3}$, tanto pré-queimadas a $1000^{\circ} \mathrm{C}$, quanto a $1250^{\circ} \mathrm{C}$. Antes do ensaio, as amostras foram mantidas por $1 \mathrm{~h}$ na temperatura de teste para garantir o equilíbrio térmico. A distância entre apoios foi de $125 \mathrm{~mm}$, utilizando-se diâmetro do rolete de $5 \mathrm{~mm}$ e taxa de carregamento de $0,750 \mathrm{kN} / \mathrm{min}$. O equipamento usado foi um Netzsch HMoR modelo 422.

O módulo de ruptura por flexão em três pontos na

Tabela I - Os ensaios foram divididos em três grupos: imprescindíveis, necessários e eventuais, segundo a representatividade das solicitações que ocorrem no revestimento em operação.

[Table I - The tests were divided in three groups: indispensable, necessary and occasional, according to the importance of the solicitations of the refractory lining under operation.]

\begin{tabular}{|c|c|c|c|c|c|}
\hline \multirow{6}{*}{ 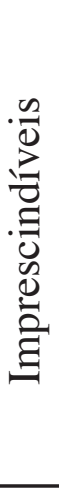 } & Ensaios & Normas & $\begin{array}{l}\text { Temp. de trat. } \\
\text { térmico prévio }\end{array}$ & Temp. dos ensaios & $\mathrm{N}^{\circ} \mathrm{CPs} / \mathrm{Dimensões}$ \\
\hline & $\begin{array}{c}\text { Resist. a Erosão na } \\
\mathrm{T}_{\mathrm{amb}} \\
\end{array}$ & ASTM C704 & $\begin{array}{c}600,800, \\
1000,1250^{\circ} \mathrm{C} \\
\end{array}$ & $25^{\circ} \mathrm{C}$ & $4 \times(114 \times 114 \times 30 \mathrm{~mm})$ \\
\hline & $\begin{array}{c}\text { Resist. a Fadiga } \\
\text { Térm. ao ar } \\
\end{array}$ & ASTM C1171 & $1000,1250^{\circ} \mathrm{C}$ & $1000^{\circ} \mathrm{C}$ & $5 \times(152 \times 25 \times 25 \mathrm{~mm})$ \\
\hline & $\begin{array}{l}\text { Mod. Rup. a Quente } \\
\text { por F3P }\end{array}$ & ASTM C583 & $1000,1250^{\circ} \mathrm{C}$ & $1000,1250^{\circ} \mathrm{C}$ & $5 \times(150 \times 25 \times 25 \mathrm{~mm})$ \\
\hline & $\begin{array}{l}\text { Mod. Rup. na } \\
\mathrm{T}_{\mathrm{amb}} \text { por F3P }\end{array}$ & ASTM C133 & $1000,1250^{\circ} \mathrm{C}$ & $25^{\circ} \mathrm{C}$ & $5 \times(288 \times 51 \times 51 \mathrm{~mm})$ \\
\hline & Porosidade Aparente & ASTM C20 & $100,1000,1250{ }^{\circ} \mathrm{C}$ & $25^{\circ} \mathrm{C}$ & $5 \times(114 \times 51 \times 51 \mathrm{~mm})$ \\
\hline \multirow{6}{*}{ 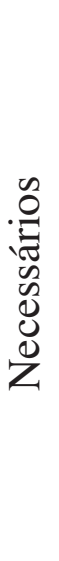 } & $\begin{array}{l}\text { Resist. a Compres. } \\
\text { na } \mathrm{T}_{\mathrm{amb}}\end{array}$ & ASTM C133 & $\begin{array}{l}100,600,800, \\
1000,1250^{\circ} \mathrm{C}\end{array}$ & $25^{\circ} \mathrm{C}$ & $5 \mathrm{x}(\varnothing 50 \times 50 \mathrm{~mm})$ \\
\hline & $\begin{array}{c}\text { Coeficiente de } \\
\text { Expansão }\end{array}$ & DIN 51053 & $1250^{\circ} \mathrm{C}$ & $\begin{array}{l}1250^{\circ} \mathrm{C} / \\
0,02 \mathrm{MPa}\end{array}$ & $\begin{array}{c}2 \times(\varnothing 50 \times 50 \mathrm{~mm}) \\
\text { (furo passante } \varnothing 12 \mathrm{~mm} \text { ) }\end{array}$ \\
\hline & $\begin{array}{c}\text { Sinterabilidade sob } \\
\text { Carga }\end{array}$ & DIN 51053 & $500{ }^{\circ} \mathrm{C}$ & $\begin{array}{l}1250^{\circ} \mathrm{C} / \\
0,20 \mathrm{MPa}\end{array}$ & $\begin{array}{c}2 \times(\varnothing 50 \times 50 \mathrm{~mm}) \\
\text { (furo passante } \varnothing 12 \mathrm{~mm} \text { ) }\end{array}$ \\
\hline & $\begin{array}{c}\text { Refratar. sob Carga } \\
{\left[\mathrm{T}_{0.5 \%}\right]} \\
\end{array}$ & DIN 51053 & $1250^{\circ} \mathrm{C}$ & $\begin{array}{l}1320{ }^{\circ} \mathrm{C} / \\
0,20 \mathrm{MPa} \\
\end{array}$ & $\begin{array}{c}2 \times(\varnothing 50 \times 50 \mathrm{~mm}) \\
\text { (furo passante } \varnothing 12 \mathrm{~mm} \text { ) }\end{array}$ \\
\hline & $\begin{array}{l}\text { Deformação por } \\
\text { Fluência }\end{array}$ & DIN 51053 & $1000,1250^{\circ} \mathrm{C}$ & $\begin{array}{c}1000,1250^{\circ} \mathrm{C} \\
48 \mathrm{~h} / 0,20 \mathrm{MPa}\end{array}$ & $\begin{array}{c}2 \times(\varnothing 50 \times 50 \mathrm{~mm}) \\
\text { (furo passante } \varnothing 12 \mathrm{~mm} \text { ) }\end{array}$ \\
\hline & $\begin{array}{c}\text { Eventual } \\
\text { Condutividade } \\
\text { Térmica }\end{array}$ & ASTM C1133 & $1000,1250^{\circ} \mathrm{C}$ & $1000,1250^{\circ} \mathrm{C}$ & $3 \times(228 \times 114 \times 64 \mathrm{~mm})$ \\
\hline
\end{tabular}


temperatura ambiente foi realizado segundo a norma ASTM C133 com distância entre apoios de $178 \mathrm{~mm}$, diâmetro do rolete de $10 \mathrm{~mm}$ e taxa de carregamento de 4,42 kN/min. Para as temperaturas de 110,1000 e $1250{ }^{\circ} \mathrm{C}$, cinco amostras $228 \mathrm{x}$ $51 \times 51 \mathrm{~mm}^{3}$ foram utilizadas para a avaliação da resistência mecânica média. $\mathrm{O}$ equipamento usado foi uma máquina universal de ensaios mecânicos MTS modelo 810/TestStarII.

A determinação da porosidade aparente dos materiais em análise foi realizada segundo uma adaptação da norma ASTM C20. As amostras utilizadas foram 5 corpos 114 x 51 x $51 \mathrm{~mm}^{3}$ resultantes da fratura das barras na determinação do módulo de ruptura a frio. Os corpos foram mantidas sob vácuo $(-690 \mathrm{mmHg}$ ) imersos em água por 1,5 h antes das leituras de massas imersa e úmida. As temperaturas de tratamento térmico analisadas foram 110,1000 e $1250{ }^{\circ} \mathrm{C}$. Para as medidas, utilizou-se uma balança digital Ohaus modelo Precision Plus 4000.

Amedida de resistência a compressão uniaxial na temperatura ambiente foi realizada em uma máquina universal de ensaios mecânicos Instron modelo 5500 com taxa de deslocamento de $1,30 \mathrm{~mm} / \mathrm{min}$, seguindo a norma ASTM C133. Para cada uma das temperaturas selecionadas $\left(110,600,800,1000\right.$ e $\left.1250{ }^{\circ} \mathrm{C}\right)$, cinco amostras cilíndricas $50 \mathrm{~mm}$ x $50 \mathrm{~mm}$ foram ensaiadas.

O coeficiente de expansão, sinterabilidade sob carga, a temperatura de refratariedade e a fluência seguiram a norma DIN 51053 e foram realizados em duplicata com amostras cilíndricas $\varnothing 50$ x 50 mm em um equipamento Netzsch RUL modelo 421.

A determinação do coeficiente de expansão com amostras pré-queimadas a $1250^{\circ} \mathrm{C}$ foi conduzido com taxa de aquecimento de $5^{\circ} \mathrm{C} / \mathrm{min}$ até $1250{ }^{\circ} \mathrm{C}$ sob $0,02 \mathrm{MPa}$ ao ar. A sinterabilidade sob carga foi realizada com amostras calcinadas a $500{ }^{\circ} \mathrm{C}$ com aquecimento a $5{ }^{\circ} \mathrm{C} / \mathrm{min}$ até $1320{ }^{\circ} \mathrm{C}$ sob $0,20 \mathrm{MPa}$ ao ar.

A temperatura de refratariedade $\left(\mathrm{T}_{0,5 \%}\right)$ foi determinada com taxa de aquecimento de $5{ }^{\circ} \mathrm{C} / \mathrm{min}$ até $1320^{\circ} \mathrm{C}$ sob 0,20 $\mathrm{MPa}$, em amostras pré-queimadas a $1250{ }^{\circ} \mathrm{C}$. Os testes de fluência foram realizados em amostras pré-queimadas a $1000 \mathrm{e}$ $1250^{\circ} \mathrm{C}$. Utilizou-se uma taxa de aquecimento de $5^{\circ} \mathrm{C} / \mathrm{min}$, sob uma carga de $0,20 \mathrm{MPa}$, com isoterma de $48 \mathrm{~h}$ a $1000^{\circ} \mathrm{C}$ para amostras já queimadas a $1000^{\circ} \mathrm{C}$, e a $1250^{\circ} \mathrm{C}$ para amostras queimadas nesta mesma temperatura.

A condutividade térmica foi avaliada nas temperaturas de queima das amostras, 1000 e $1250{ }^{\circ} \mathrm{C}$, segundo a norma ASTM C1113. Para cada medida foram necessários 3 tijolos 228 × $114 \times 64 \mathrm{~mm}^{3}$. O equipamento utilizado foi Netzsch TCT modelo 426.

\section{RESULTADOS E DISCUSSÃO}

\section{Ensaios imprescindíveis}

Determinação da resistência a erosão na temperatura ambiente

Antes de cada série de 16 amostras, tanto do material AWS quanto do concreto BDG foram realizados ensaios de erosão em corpos de vidro soda-cal para aferir o equipamento e garantir que ambos os materiais fossem ensaiados sobre as mesmas condições. A diferença entre os valores foi de $0,2 \mathrm{~cm}^{3}$, bem inferior ao previsto por norma $\left(0,5 \mathrm{~cm}^{3}\right)$, indicando boa reprodutibilidade.

As amostras do material AWS foram enviadas após projeção e corte, sem a realização de retífica. Os corpos de prova deste concreto apresentaram acabamento superficial ruim, porém mais realista, já que melhor representam o material após a projeção. Tal característica pode ter prejudicado seu desempenho já que a presença de defeitos superficiais funciona como catalisador para nucleação de trincas, degradando as propriedades mecânicas.

Todos os corpos BDG tiveram as faces integralmente retificadas, apresentando um bom acabamento superficial. Mas, após a erosão foram evidenciadas trincas em materiais antes, aparentemente, sem defeitos. Neste material também pôde ser observada grande quantidade de agregados de cerca de $10 \mathrm{~mm}$. Estes grãos de elevado diâmetro devem tornar a aplicação por dry-gunning mais trabalhosa, podendo ocorrer entupimentos da tubulação durante o transporte pneumático.

O acabamento de baixa qualidade do AWS é evidenciado pelos elevados valores de desvio padrão apresentados no gráfico de volume erodido (Fig. 3), pois corpos que, teoricamente, deveriam estar sob condições idênticas, apresentavam distintas quantidades de defeitos.

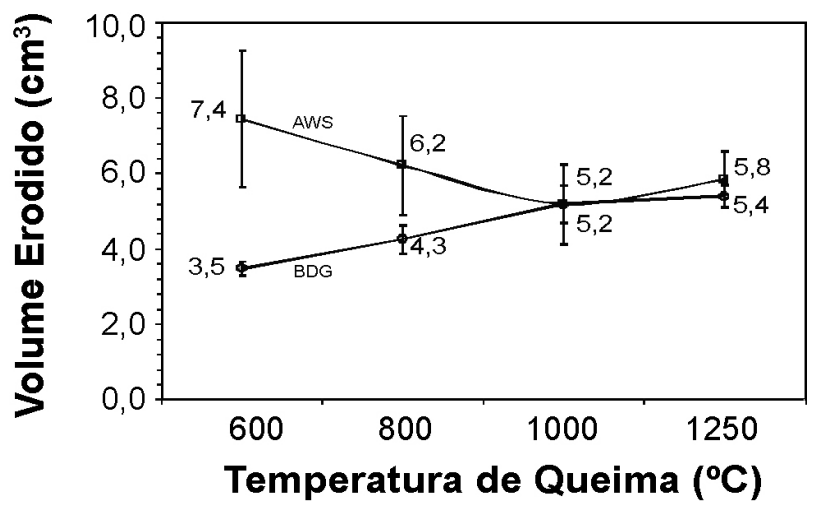

Figura 3: Volume erodido em função da temperatura de queima para os materiais AWS e BDG.

[Figure 3: Erosion volume in function of the firing temperature for the materials $A W S$ and $B D G$.]

A redução na resistência à erosão do concreto $\mathrm{BDG}$ com o aumento da temperatura pode estar relacionada com a sua composição química. O material BDG apresentou desempenho superior ao concreto AWS em temperaturas até $800^{\circ} \mathrm{C}$. Já para corpos queimados a $1000{ }^{\circ} \mathrm{C}$ os resultados foram semelhantes, assim como a $1250{ }^{\circ} \mathrm{C}$, considerando-se o desvio padrão.

É importante lembrar que materiais com elevado teor de fases vítreas apresentam melhores desempenhos mecânicos na temperatura ambiente, mas o líquido formado em altas temperaturas reduz as propriedades termomecânicas.

\section{Fadiga térmica ao ar}

Mesmo com acabamento de menor qualidade, evidenciado novamente pelo maior desvio padrão, o concreto 
AWS apresentou maiores módulos de ruptura em amostras pré-queimadas a $1000{ }^{\circ} \mathrm{C}$ sem choque térmico $(10,1 \mathrm{MPa}) \mathrm{e}$ submetidas a fadiga térmica (5,8 $\mathrm{MPa})$ ao ar (Fig. 4).

No entanto, o material AWS também obteve uma maior queda porcentual em seu módulo de ruptura (42\%), em relação ao BDG (23\%), o que pode indicar uma microestrutura mais susceptível ao dano por choque térmico ou que a menor qualidade do acabamento superficial também está influenciando a resistência a fadiga térmica.

Deve ser destacado que o mais importante nesta avaliação não é a queda porcentual, mas sim o valor absoluto da resistência mecânica residual após os 10 ciclos de choque térmico.

Ensaios adicionais revelaram que a porosidade e a densidade aparentes não foram alteradas significativamente após os ensaios de fadiga térmica ao ar nas amostras do concreto AWS. Por isto, a maior queda porcentual na resistência mecânica deve estar associada ao aumento no tamanho dos defeitos críticos já existentes, devido às tensões

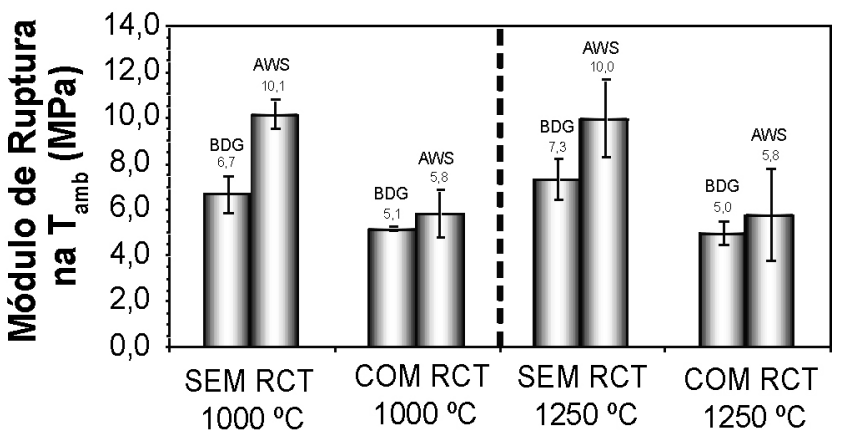

Figura 4: Módulo de ruptura por flexão em três pontos para amostras dos materiais AWS e BDG queimadas a 1000 e $1250{ }^{\circ} \mathrm{C}$ antes e após o teste de resistência ao choque térmico ao ar.

[Figure 4: Three points bending for the materials AWS and BDG fired at 1000 and $1250{ }^{\circ} \mathrm{C}$ before and after the thermal shock resistance test in air.]

termomecânicas, e não ao microtrincamento.

O material AWS pré-queimado a $1250^{\circ} \mathrm{C}$ obteve valores semelhantes de módulo de ruptura aos observados para os testes com pré-queima de $1000{ }^{\circ} \mathrm{C}$. A queda porcentual no módulo antes e após a fadiga térmica foi, novamente, superior $(42 \%)$ em relação ao BDG $(31 \%)$.

Em virtude da constância de propriedades mesmo com a variação nas condições de queima, e aos valores das resistências mecânicas a $1000{ }^{\circ} \mathrm{C}$ e $1250{ }^{\circ} \mathrm{C}$ serem, respectivamente, $37 \%$ e $16 \%$ mais elevados, pode-se considerar o material AWS mais resistente ao dano por choque térmico cíclico ao ar $\left(\Delta \mathrm{T}=1000{ }^{\circ} \mathrm{C}\right)$, mesmo com acabamento superficial inferior, porém, mais realista.

\section{Módulo de ruptura a quente}

O módulo de ruptura a quente (MRQ), realizado a $1000{ }^{\circ} \mathrm{C}$ no concreto AWS pré-queimado a $1000{ }^{\circ} \mathrm{C}$, foi significativamente mais elevado (131\%) que o apresentado pelo material BDG. Apesar disto, o elevado desvio evidencia o acabamento superficial de menor qualidade das amostras AWS (Fig. 5). A $1250{ }^{\circ} \mathrm{C}$, houve uma queda intensa nos valores de módulo de ruptura a quente, tanto do AWS (26 para $9 \mathrm{MPa}$ ) quanto do BDG (11 para $5 \mathrm{MPa}$ ), mas ainda assim, o concreto AWS pré-queimado a $1250{ }^{\circ} \mathrm{C}$ obteve uma resistência cerca de $73 \%$ superior que o material BDG. Estes resultados permitem estimar que a resistência a erosão a quente do concreto AWS deve ser superior ao do concreto BDG, uma vez que a literatura [6] aponta uma boa correlação entre o MRQ e o ensaio de erosão a quente.

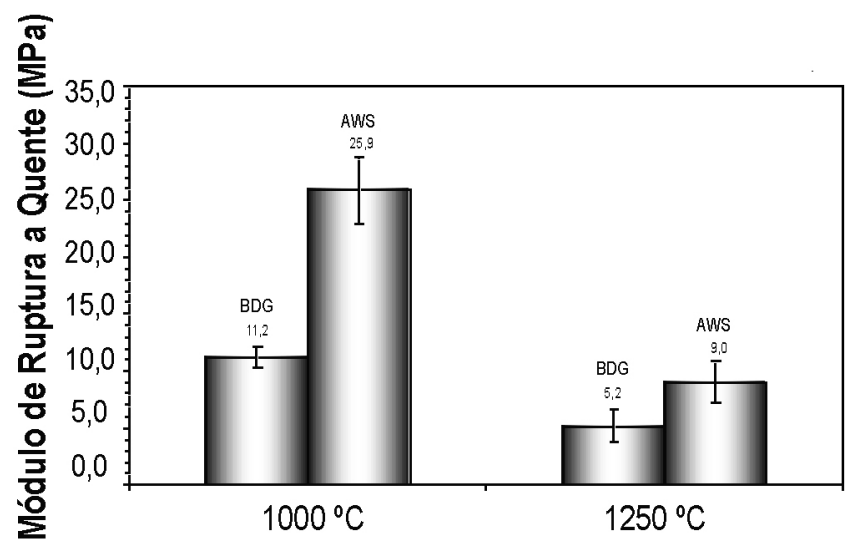

Figura 5: Módulo de ruptura a quente $\left(1000\right.$ e $\left.1250^{\circ} \mathrm{C}\right)$ para os materiais AWS e BDG.

[Figure 5: Hot modulus of rupture at 1000 and $1250{ }^{\circ} \mathrm{C}$ for materials AWS and BDG.]

Módulo de ruptura por flexão em 3 pontos na temperatura ambiente

Apesar do concreto BDG apresentar um módulo de ruptura $59 \%$ superior após secagem a $110{ }^{\circ} \mathrm{C} / 24 \mathrm{~h}$, em relação ao AWS, ele cai sensivelmente (137\%) e se mantêm constante próximo de $6,8 \mathrm{MPa}$ para as temperaturas de $1000{ }^{\circ} \mathrm{C}$ e $1250{ }^{\circ} \mathrm{C}$ (Tabela II). Esta elevada resistência mecânica após secagem pode ser um indicativo da grande quantidade de ligante presente no material, o que reduz a refratariedade, conforme os resultados de módulo de ruptura a quente mostraram. Apesar do AWS possuir módulo de ruptura a $110{ }^{\circ} \mathrm{C}$ mais baixo que o $\mathrm{BDG}$, este resultado não é tão significativo para a operação do calcinador, pois estes materiais serão aplicados por projeção e não necessitarão de elevadas resistências mecânicas para resistirem à etapa de desmoldagem. Embora a resistência mecânica mais elevada do material BDG na temperatura ambiente possa indicar uma melhor capacidade do material em suportar maiores espessuras projetadas, deve-se ter cuidado no dimensionamento do revestimento, pois quanto maior a espessura, maior será a taxa de fluência quando a resistência mecânica for reduzida na decomposição do ligante hidráulico. 
O material AWS apresentou propriedades mecânicas a frio crescentes com o aumento da temperatura de queima, sendo que o módulo entre 1000 e $1250{ }^{\circ} \mathrm{C}$ também atingiu um patamar, mas em torno de $11,5 \mathrm{MPa}$, cerca de $70 \%$ mais elevado que o BDG.

É interessante notar que se for realizada uma correlação do módulo de ruptura sob três pontos com a resistência a erosão, ambos na temperatura ambiente, o material AWS deveria apresentar menores volumes erodidos que o material BDG. Porém, deve ser ressaltado que o acabamento superficial das amostras do material AWS para erosão estava bem inferior que o observado nos corpos usados para a determinação do módulo de ruptura.

\section{Determinação da porosidade aparente}

Apesar do bom acabamento superficial das amostras BDG utilizadas para as medidas de porosidade, como também havia uma superfície interna de fratura, foi possível verificar que o material aplicado por dry-gunning apresenta uma maior porosidade, em relação ao AWS, projetado por wet-shotcrete, independente da condição de tratamento térmico $\left(110,1000,1250{ }^{\circ} \mathrm{C}\right)$, conforme a Tabela II. Este resultado não concorda diretamente com o elevado valor de módulo de ruptura do material BDG, mas pode ser mais um indício que este material apresenta grande quantidade de ligante, ou até mesmo dois ligantes combinados.

Tabela II - Módulo de ruptura por flexão em três pontos e porosidade aparente para amostras dos materiais AWS e BDG após secagem a $110^{\circ} \mathrm{C}$, e queimadas a 1000 e $1250^{\circ} \mathrm{C}$.

[Table II - Three point bending and apparent porosity for samples of the materials $A W S$ and $B D G$ after drying at $110^{\circ} \mathrm{C}$, and fired at $1000^{\circ} \mathrm{C}$ and $1250^{\circ} \mathrm{C}$.]

\begin{tabular}{lccccc}
\hline & & $\begin{array}{c}\text { Mód. Rup. } \\
\text { (MPa) }\end{array}$ & $\begin{array}{c}\text { Desv } \\
\text { Pad. }\end{array}$ & $\begin{array}{c}\text { Por. Ap. } \\
(\%)\end{array}$ & $\begin{array}{c}\text { Desv. } \\
\text { Pad. }\end{array}$ \\
\hline $110^{\circ} \mathrm{C}$ & BDG & 16,1 & 0,9 & 16,1 & 0,5 \\
& AWS & 10,1 & 1,1 & 14,1 & 1,0 \\
\hline $1000^{\circ} \mathrm{C}$ & BDG & 6,9 & 0,5 & 19,9 & 1,2 \\
& AWS & 11,5 & 1,2 & 19,8 & 1,2 \\
\hline $1250^{\circ} \mathrm{C}$ & BDG & 6,7 & 0,7 & 20,9 & 0,9 \\
& AWS & 11,8 & 1,3 & 19,6 & 0,5 \\
\hline
\end{tabular}

\section{Ensaios necessários}

\footnotetext{
Resistência à compressão uniaxial na temperatura ambiente

A tendência apresentada pelos resultados de resistência a compressão (Fig. 6), em especial considerando as temperaturas de $110,1000 \mathrm{e} 1250^{\circ} \mathrm{C}$, foi semelhante à apresentada pelo ensaio de módulo de ruptura por flexão em três pontos.
}

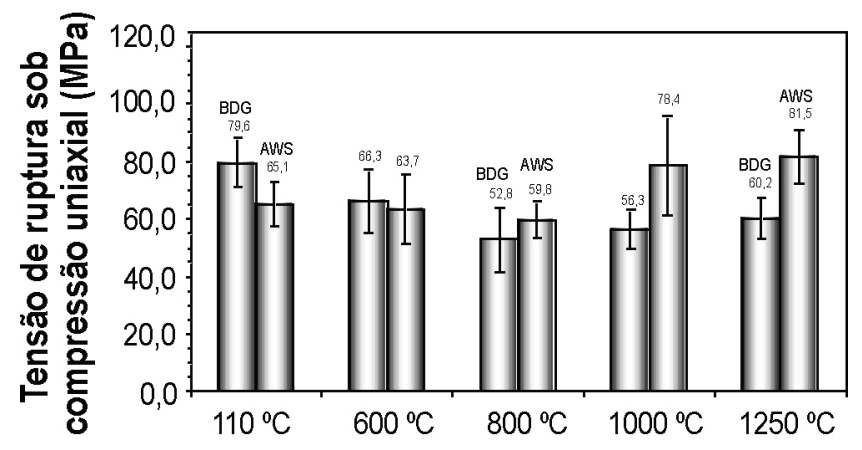

Figura 6: Módulo de ruptura por compressão uniaxial para os materiais AWS e BDG.

[Figure 6: Cold crushing modulus of rupture for materials AWS and $B D G$.]

Nota-se que o material BDG apresenta um elevado valor de módulo de ruptura $(79,6 \mathrm{MPa})$ após secagem $\left(110{ }^{\circ} \mathrm{C}\right)$, mas que se estabiliza em valores inferiores $(\sim 60 \mathrm{MPa})$ para as temperaturas mais elevadas de queima $\left(1000\right.$ e $\left.1250{ }^{\circ} \mathrm{C}\right)$. Isto reforça a teoria de grande quantidade de ligante a verde. No caso do material AWS, semelhantemente aos resultados de resistência a flexão, há uma evolução das propriedades com o aumento da temperatura de queima (Fig. 6). O valor inicial da resistência a compressão é de $65,1 \mathrm{MPa}\left(110^{\circ} \mathrm{C}\right)$, e alcança um patamar em torno de $80 \mathrm{MPa}$ nas temperaturas mais elevadas de queima $\left(1000\right.$ e $\left.1250{ }^{\circ} \mathrm{C}\right)$. fluência

Expansão, sinterabilidade, refratariedade sob carga e

$\mathrm{O}$ coeficiente de expansão térmica linear médio dos materiais AWS $\left(6,0 \times 10^{-6}{ }^{\circ} \mathrm{C}^{-1}\right)$ e BDG $\left(6,3 \times 10^{-6}{ }^{\circ} \mathrm{C}^{-1}\right)$ podem ser considerados na faixa esperada para concretos aluminosilicosos da classe $70 \%$ de $\mathrm{Al}_{2} \mathrm{O}_{3}$. O valor ligeiramente inferior para o concreto AWS pode estar relacionado com fases de menor expansão em sua composição (p.e. mulita) e que auxiliam na resistência ao choque térmico. Foi observado que o perfil de sinterização sob carga de ambos os concretos avaliados são bastante semelhantes até $1200^{\circ} \mathrm{C}$. Na região entre 700 e $900{ }^{\circ} \mathrm{C}$ foram observadas deformações similares nos materiais AWS e BDG, atribuídas a saída de água estrutural dos ligantes e das fases inorgânicas (por exemplo argila).

Observa-se na Fig. 7 que a retração na faixa entre 700 e $900{ }^{\circ} \mathrm{C}$ ocorre mais intensamente nos corpos BDG que nas amostras AWS. Isto é mais um indicativo do elevado teor de cimento presente no concreto BDG, e que se decompõe acima da temperatura de pré-calcinação $\left(500^{\circ} \mathrm{C}\right)$.

Em temperaturas superiores a $1200{ }^{\circ} \mathrm{C}$ pode ser observado um padrão de deformação atribuído a sinterização sob carga, que difere significativamente entre os concretos. Os segmentos de sinterização para o AWS indicam uma deformação final $62 \%$ menor (-1,15\%), em relação ao material $\operatorname{BDG}(-1,87 \%)$. Adicionalmente, nota-se que próximo à temperatura máxima de ensaio $\left(1320^{\circ} \mathrm{C}\right)$ o concreto AWS apresenta uma leve inflexão, indicando uma atenuação na 


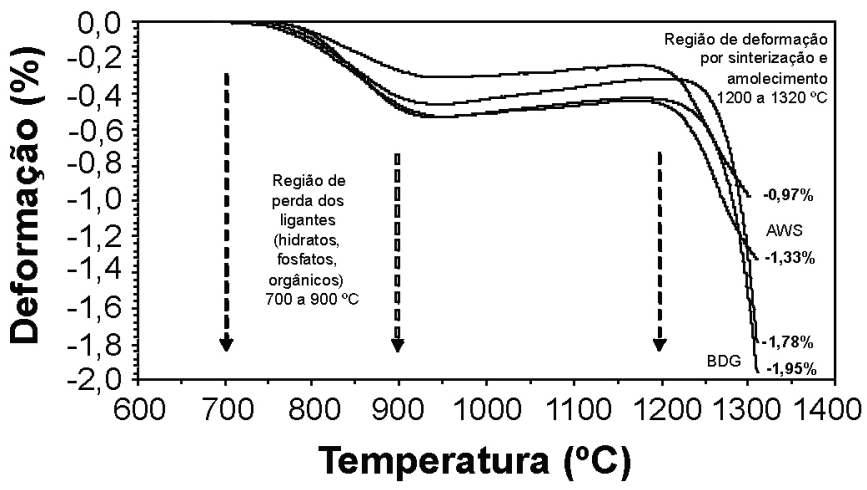

Figura 7: Segmentos de deformação das curvas obtidas no ensaio de sinterabilidade sob carga.

[Figure 7: Deformation curves obtained on the sintering under load tests.]

deformação para temperaturas mais elevadas, possivelmente atribuída a mulitização in-situ. Para o material BDG notase, possivelmente, a presença de grande quantidade de fases líquidas acima de $1250^{\circ} \mathrm{C}$, uma vez que a tendência à deformação é muito acentuada.

Conforme pode ser observado na Fig. 8, a temperatura de amolecimento $\left(\mathrm{T}_{\text {amol }}\right)$ do concreto BDG $\left(1159{ }^{\circ} \mathrm{C}\right)$ é significativamente inferior à apresentada pelo material AWS $\left(1253^{\circ} \mathrm{C}\right)$, sendo que a temperatura de refratariedade $\left(\mathrm{T}_{0,5 \%}\right)$ no material BDG $\left(1299^{\circ} \mathrm{C}\right)$ é pouco acima da $\mathrm{T}_{\text {amol }}$ do concreto AWS, e a $\mathrm{T}_{05 \%}$ deste é bem acima da temperatura máxima de ensaio $\left(1320^{\circ} \mathrm{C}\right)$. A expansão máxima do material AWS foi de $0,58 \%$ no intervalo entre 100 e $1253^{\circ} \mathrm{C}$, enquanto o BDG teve $0,53 \%$ de expansão entre 100 e $1159^{\circ} \mathrm{C}$.

Esta menor refratariedade do material BDG pode ser um reflexo do grande teor de fases fundentes na sua formulação, e concorda com os menores valores de módulo de ruptura a quente, e possivelmente, de resistência a erosão a quente.

Os ensaios de fluência conduzidos a $1000{ }^{\circ} \mathrm{C} / 48 \mathrm{~h}$ (Fig. 9) mostraram que a amostra BDG (-0,036\%) apresentou uma deformação cerca de três vezes menor que o concreto AWS $(-0,107 \%)$. Mas, ambos os materiais apresentaram deformação a $1000{ }^{\circ} \mathrm{C}$ em níveis considerados baixos e adequados.

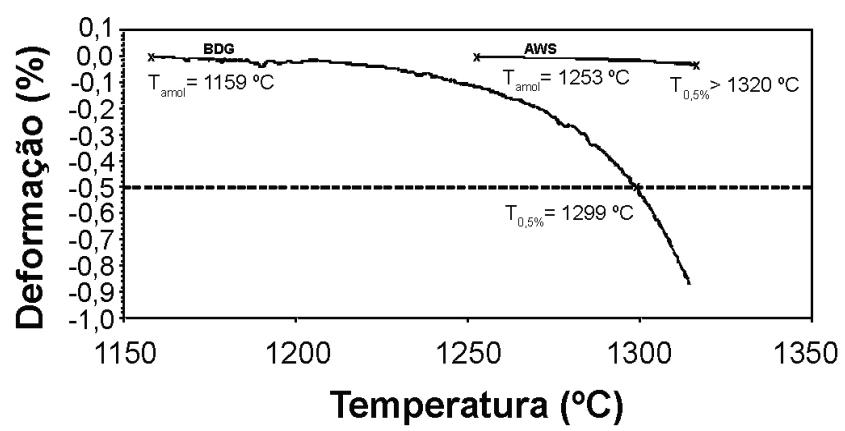

Figura 8: Segmentos de deformação após amolecimento obtidos nas curvas de refratariedade sob carga para os materiais AWS e BDG.

[Figure 8: Deformation curves for refractoriness under load tests.]

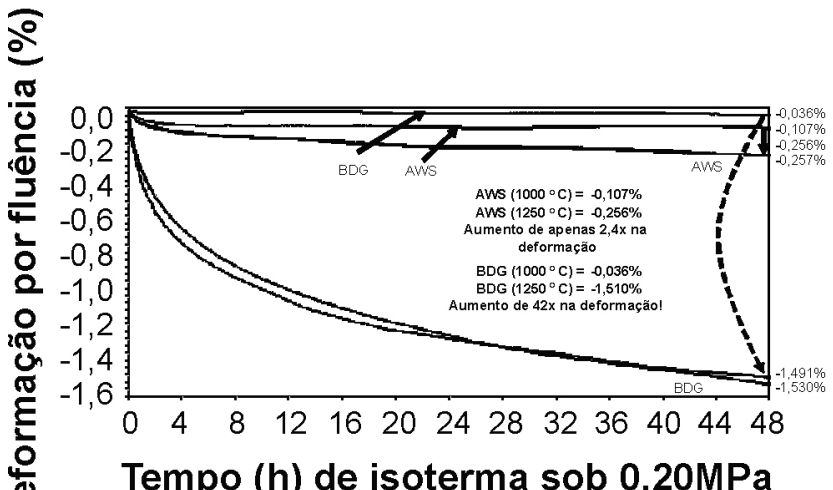

Figura 9: Curvas de deformação por fluência a 1000 e $1250{ }^{\circ} \mathrm{C} / 48 \mathrm{~h}$ para o AWS e o BDG.

[Figure 9: Creep curves at 1000 and $1250{ }^{\circ} \mathrm{C} / 48 \mathrm{~h}$ for $\mathrm{AWS}$ and BDG samples.]

No entanto, quando se compara a deformação após $1000{ }^{\circ} \mathrm{C} / 48 \mathrm{~h}$ com a obtida a $1250{ }^{\circ} \mathrm{C}$, observa-se uma maior regularidade do material AWS, o qual aumentou apenas 2,4 vezes sua deformação, em relação ao BDG, que teve aumento de mais de 40 vezes, isto é, $-0,036 \%$ a $1000{ }^{\circ} \mathrm{C}$ e $-1.510 \%$ para $1250{ }^{\circ} \mathrm{C}$.

Os resultados de fluência obtidos a $1250{ }^{\circ} \mathrm{C} / 48 \mathrm{~h}$ concordam com a refratariedade sob carga e o módulo de ruptura a quente apresentados anteriormente. Observou-se na Fig. 9 que o concreto AWS apresentou uma deformação média final cerca de 6 vezes menor $(-0,26 \%)$ em relação ao concreto BDG (-1,51\%), indicando uma superior refratariedade.

A boa reprodutibilidade dos ensaios de fluência apresentada pelos corpos da composição AWS também indica que, apesar do acabamento superficial de menor qualidade, a qual pode ter interferido no resultado de vários testes (p.e. erosão, módulos de ruptura a frio e a quente, resistência ao choque térmico), a sua formulação parece ser mais adequada que o material BDG.

Uma composição mineralógica mais "engenheirada" leva a uma homogenidade microestrutural mais elevada, e que pode ser indiretamente detectada por testes que consideram todo o volume do material, tais como a refratariedade sob carga e a fluência, tendo o acabamento superficial uma menor influência.

A menor refratariedade apresentada pelo concreto BDG, em relação ao AWS, é possivelmente um reflexo do grande teor de cimento na formulação $(>3 \%$-p $\mathrm{CaO})$. Isto possibilita a formação de fases de menor ponto de fusão no sistema $\mathrm{Al}_{2} \mathrm{O}_{3}-\mathrm{SiO}_{2}-\mathrm{CaO}$, conforme a reação: $\mathrm{Al}_{2} \mathrm{O}_{3}+$ líquido $\rightarrow$ Anortita + Guelenita $+\mathrm{Al}_{2} \mathrm{O}_{3}$

Isto pôde ser comprovado pela elevada intensidade dos picos de difração de raios X para a anortita (menor temperatura de fusão) que o material BDG apresentou. Apesar do concreto AWS também possuir anortita, os picos foram menores e se igualam à quantidade de guelenita, fase 
de maior ponto de fusão. Adicionalmente, a resistência ao choque térmico mais elevada do material AWS pode ser atribuída à maior presença de mulita, enquanto o BDG possui mais alumina, fase de menor resistência a fadiga térmica. Ambas as formulações têm sílica livre na forma de tridimita.

\section{Ensaio eventual}

\section{Condutividade térmica}

Os resultados de condutividade térmica obtidos para os materiais AWS $\left(1,91 \mathrm{~W} / \mathrm{mK}\right.$ a $1000^{\circ} \mathrm{C}$ e $2,13 \mathrm{~W} / \mathrm{mK}$ a 1250 $\left.{ }^{\circ} \mathrm{C}\right)$ e BDG $\left(2,04 \mathrm{~W} / \mathrm{mK}\right.$ a $1000{ }^{\circ} \mathrm{C}$ e $2,03 \mathrm{~W} / \mathrm{mK}$ a $\left.1250{ }^{\circ} \mathrm{C}\right)$ estão de acordo com valores encontrados na literatura para materiais alumino-silicosos com 70\%-p $\mathrm{Al}_{2} \mathrm{O}_{3}(1,8$ a 2,2 W/ $\mathrm{mK}$ entre 600 e $1200{ }^{\circ} \mathrm{C}$ ). Estes valores de condutividade auxiliam na resistência ao choque térmico, porém, requerem bom isolamento térmico para evitar perdas excessivas de calor.

Adicionalmente, foi realizada na amostra AWS sinterizada a $1250{ }^{\circ} \mathrm{C}$ uma medida de condutividade a 1000 ${ }^{\circ} \mathrm{C}$ para podermos comparar com o valor obtido no material pré-queimado e ensaiado a $1000{ }^{\circ} \mathrm{C}$. O resultado revela que a maior densificação do concreto após queima a $1250{ }^{\circ} \mathrm{C}$ conduz a valores $38 \%$ superiores de condutividade $(1,91$ para $2,64 \mathrm{~W} / \mathrm{mK}$ ), indicando uma condição mais severa de análise e que permite reduzir a quantidade de ensaios de condutividade térmica.

\section{CONCLUSÕES}

O embasamento técnico e científico utilizado neste trabalho pôde orientar o desenvolvimento, a seleção e a aplicação de novas cerâmicas refratárias para o revestimento interno de calcinadores de alumina com o objetivo de elevar a vida útil, a disponibilidade e a confiabilidade das unidades de calcinação.

Em virtude da constância de propriedades e dos valores mais elevados de módulo de ruptura, pode-se considerar o material AWS mais resistente ao dano por choque térmico cíclico mesmo com acabamento superficial inferior.

A resistência a flexão a quente realizada a $1000{ }^{\circ} \mathrm{C}$ e a $1250{ }^{\circ} \mathrm{C}$ no concreto AWS mostrou valores superiores aos apresentados pelo material BDG, mesmo com acabamento superficial de menor qualidade, o que indica uma maior resistência à erosão a quente do concreto AWS.

Embora o perfil de sinterização sob carga de ambos os concretos avaliados seja semelhante, a retração na faixa entre 700 e $900{ }^{\circ} \mathrm{C}$, decorrente da decomposição dos ligantes, ocorre mais intensamente nos corpos BDG do que nas amostras AWS, indicando um maior teor de cimento no concreto BDG.

Em temperaturas superiores a $1200{ }^{\circ} \mathrm{C}$ o padrão de deformação atribuído a sinterização sob carga evidencia que a deformação final do AWS é 62\% inferior em relação ao material BDG. Além disto, o BDG apresenta baixas temperaturas de amolecimento e de refratariedade, indicando uma inferior resistência à erosão a quente, conforme relatado [2].

A boa reprodutibilidade dos ensaios de fluência apresentada pelo AWS indica que apesar do acabamento superficial de menor qualidade, o qual interferiu em vários testes, a sua formulação é mais adequada que a do material BDG. Isto, pois uma composição mineralógica mais "engenheirada" leva a uma superior homogenidade microestrutural, e pode ser indiretamente detectada por testes que consideram menos os defeitos na superfície, e avaliam o volume do material como um todo, tais como a refratariedade sob carga e a fluência.

Baseado nos resultados obtidos neste trabalho, o concreto AWS aplicado por wet-shotcrete possui o melhor desempenho de propriedades a 1000 e $1250{ }^{\circ} \mathrm{C}$, faixa de temperatura de trabalho do calcinador de alumina. Apesar dos corpos não terem sido retificados, as propriedades termomecânicas foram superiores ao material BDG. Caso as amostras AWS tivessem um melhor acabamento, poderia ser verificada uma melhoria ainda maior em suas propriedades. No entanto, se o acabamento superficial do concreto BDG fosse mais realista, suas propriedades seriam ainda inferiores às obtidas.

\section{AGRADECIMENTOS}

Os autores agradecem a colaboração técnica de Vito A. A. Santos, Vânia R. Salvini, Paulo T. Tiba e José L. Santos; ao GEMM/UFSCar, CAPES e Alcoa Alumínio S.A. pelo suporte oferecido e às empresas que forneceram os materiais para os testes.

\section{REFERÊNCIAS}

[1] G. E. Snyder, S. Ehrlich, Refractories in fluidized-bed combustors, Am. Ceram. Soc. Bull. 73 (1994) 62-65.

[2] A. Pinoncely, K. Tsouria, FCB flash calciner technology: ten years performances, Light Metals (1995) 113-120.

[3] G. P. Le Page, M. O. Tade, R. J. Stone, Comparative evaluation of advanced process control techniques for alumina flash calciners, J. Proc. Cont. 8 (1998) 287-298.

[4] B. Whittington, D. Ilievski, Determination of the gibbsite dehydration reaction pathway at conditions relevant to Bayer refineries, Chem. Eng. J. 98 (2004) 89-97.

[5] A. Kontopoulos, K. Krallis, E. Koukourakis, N. Denaxas, N. Kostis, A. Broussaudf, 0. Guyot, A hybrid, knowledgebased system as a process control 'tool' for improved energy efficiency in alumina calcining furnaces, Appl. Thermal Eng. 17 (1997) 935-945.

[6] D. A. A. Lopes, V. C. Pandolfelli, Revestimento refratário para unidades de calcinação de alumina em leito fluidizado: uma abordagem sistêmica, Diss. Mestrado, UFSCar (2005) 136 p.

[7] E. W. Lussky, Experience with operation of the Alcoa fluid-flash calciner, Light Metals (1980) 69-79.

[8] W. M. Fish, Alumina calcination in the fluid-flash 
calciner, TMS paper No. A74-63, Metall. Soc. AIME (1974) $10 \mathrm{p}$.

[9] J. Barrie, B. Christine, Improvements in the design and operation of alumina flash calciners, IFRF Combustion J. (2001) 200110.

[10] L. Huilin, B. Rushan, L. Wenti, L. Binxi, Y. Lidan, Computations of a circulating fluidized-bed boiler with wide particle size distributions, Ind. Eng. Chem. Res. 39 (2000) 3212-3220
[11] V. A. A. Santos, F. A. O. Valenzuela, J. B. Gallo, V. C. Pandolfelli, Fundamentos e testes preliminares de resistência a erosão de concretos refratários, Cerâmica 52 (2006) 128 135.

[12] A. Di Renzo, F. P. Di Maio, Homogeneous and bubbling fluidization regimes in DEM-CFB simulations: hydrodynamic stability of gas and liquid fluidized beds, Chem. Eng. Sci. 62 (2007) 116-130.

(Rec. 18/04/2007, Ac. 17/08/2007) 